\title{
Resolución de problemas matemáticos en la educación media costarricense: un estudio acerca de la actitud
}

Luis Gerardo Meza-Cascante', Zuleyka Suárez-Valdés-Ayala², Evelyn

Agüero-Calvo ${ }^{3}$, Instituto Tecnológico de Costa Rica

Recibido: 15 de julio, 2015.

Aprobado: 25 octubre, 2015

\section{Resumen}

En este artículo se exponen los resultados de un estudio realizado sobre de la actitud de los estudiantes de la educación media oficial costarricense hacia la resolución de problemas matemáticos. En dicho estudio, se indagó la existencia de diferencias entre hombres y mujeres y, además, se identificaron los niveles educativos con mejor actitud. La investigación se realizó con una muestra de 3271 estudiantes durante el año 2014.

Los resultados muestran que aproximadamente un $20 \%$ de los estudiantes tiene una actitud baja o muy baja hacia la resolución de problemas matemáticos, lo cual es poco favorable, y que cerca del 50\% tiene una actitud media, es decir, ni baja ni alta. También, los resultados revelan diferencias estadísticamente significativas en el nivel de actitud hacia la resolución de problemas matemáticos por sexo, donde los hombres presentan niveles mayores, aunque con un tamaño del efecto bajo.

Los hallazgos sugieren entonces que no hay diferencias en el nivel de actitud entre los estudiantes de décimo y undécimo año, y que estos niveles evidencian niveles más bajos de actitud comparados con sétimo, octavo y noveno; lo anterior resulta preocupante por cuanto los niveles menos favorables de actitud se dan precisamente en los estudiantes más próximos a elegir carrera universitaria, en donde el componente matemático es vital para la selección de opciones en ciencia y tecnología.

1 Doctor en Educación con énfasis en Investigación Educativa (UNED, Costa Rica) y Licenciado en la Enseñanza de la Matemática, Universidad Nacional, Costa Rica. Docente, investigador y Director de la Escuela de Matemática del Instituto Tecnológico de Costa Rica (ITCR) Contacto: gemeza@itcr.ac.cr.

2 Máster en educación con énfasis en Docencia Universitaria de la Universidad Nacional, Costa Rica y Licenciada en la Enseñanza de la Matemática de la Universidad Nacional, Costa Rica. Actualmente, es docente e investigadora en la Escuela de Matemática del Instituto Tecnológico de Costa Rica (ITCR). Contacto: zsuarez@itcr.ac.cr.

3 Docente e investigadora de la Escuela de Matemática, Instituto Tecnológico de Costa Rica. Se graduó como licenciada en Enseñanza de la Matemática del Instituto Tecnológico de Costa Rica y como Doctora en Educación, en la Universidad de Valencia, España. Contacto: evaguero@itcr.ac.cr.

PALABRAS CLAVE:

Actitud, resolución de problemas, problemas matemáticos, aprendizaje de la matemática, educación media, afectividad.

\section{KEY WORDS:}

Attitude, problem solving, mathematical problems, math learning, middle education, affectivity. 


\section{Abstract \\ SOLVING OF MATHEMATICAL PROBLEMS IN COSTA RICAN SECONDARY EDUCATION: A STUDY ABOUT ATTITUDE}

This paper presents the results of a study regarding Costa Rican official public high school students' attitude toward the solving of mathematical problems. In such study, the existence of differences between men and women was researched. The best educational levels with the best attitude were also identified. The research was based upon a sample of 3271 students along the year 2014.

The results show that approximately $20 \%$ of students has a low or very low attitude in regards to solving mathematical problems, which is not really favorable; $50 \%$ has a middle attitude, this is, neither low nor high. Also, the results reveal significant statistical differences in the level of attitude toward solving mathematical problems based upon sex, showing that men have the higher levels, even though the amount of the effect is low.

The findings suggest that there are no differences in the level of attitude between tenth graders and eleventh graders, being these levels the one that show the lowest attitude in comparison to the seventh, eighth and ninth grades, which becomes worrisome since the least favorable levels of attitude are precisely found in those students who will soon face the choice of a university career, where the mathematical component is vital in order to choose technology or science options.

\section{INTRODUCCIÓN}

En los procesos de enseñanza y aprendizaje de la matemática los factores cognitivos son sustantivos, pero también son importantísimos los aspectos afectivos, porque pueden condicionar la evolución y éxito de los estudiantes en esta materia (Pérez-Tyteca, 2012).

Por aspectos afectivos se entiende, de acuerdo con Mc Leod (1989, citado por Gómez-Chacón, 2000), el "extenso rango de estados de ánimo que son generalmente considerados como algo diferente de la pura cognición e incluye como componentes específicos las creencias, las actitudes y las emociones" (p. 186).

Además, de acuerdo con Gil, Blanco y Guerrero (2006), gran parte de los estudiantes percibe que el conocimiento matemático es complejo y generador de sentimientos de intranquilidad, miedo, ansiedad, inseguridad, desconcierto e incertidumbre. Además, afirman que los alumnos expresan con frecuencia sus sentimientos acerca de la matemática mediante frases como "odio las clases de matemáticas", "las matemáticas son aburridas" o "la resolución de problemas me produce ansiedad". Palabras como las anteriores (y otras similares), indican estos autores, ponen de relieve la influencia e importancia de los factores afectivos en la enseñanza y aprendizaje de la matemática.

Ashcraft y Faust (1994), citados por Núñez-Peña, Suárez-Pellicioni y Bono (2013), mostraron que los anteriores factores están relacionados también con el bajo desempeño en los cursos de matemática, y encontraron que los efectos son peores en la resolución de problemas aritméticos complejos.

Gómez-Chacón (2000) por su parte, considera que la alta frecuencia de fracasos en el aprendizaje de la matemática, en diversas edades y niveles educativos, puede ser explicada en gran parte por las actitudes negativas que tienen los aprendices, generadas por factores personales y ambientales, cuya detección sería el primer eslabón de una cadena de acciones efectivas encaminadas a eliminar esa influencia negativa.

Las actitudes hacia la matemática constituyen un campo muy valioso para la investigación en el dominio afectivo de esta asignatura (Palacios, Arias y Arias, 2014). El estudio de las actitudes de los estudiantes ha despertado el interés de los investigadores en matemática educativa, en la medida que se comprueba la insuficiencia de los planteamientos tradicionales para alcanzar los objetivos de una sociedad cada vez más exigente (Hernández, 2011).

La ansiedad en matemática ha sido objeto de estudio en los últimos años, y se ha encontrado que las personas que la padecen evitan los ambientes y las carreras que requieran el uso de este curso (Primi, Busdraghi, Tomasetto, Morsanyi y Chiesi, 2014). Estos mismos autores demuestran que las personas con mayor ansiedad matemática tienen un menor desempeño que las que tienen niveles más bajos en tareas de corte matemático. 
Además, de acuerdo con Gil, Blanco y Guerrero (2006), las mujeres muestran una actitud más negativa que los hombres y experimentan mayores niveles de ansiedad, desconfianza, inseguridad, falta de perseverancia; también señalan que este grupo siente menos curiosidad por la solución de problemas, menos sensación de fracaso y de pérdida de tiempo hacia las matemáticas y su aprendizaje, por lo que los citados investigadores concluyen que el género está asociado a ciertas actitudes y reacciones emocionales hacia la matemática.

No obstante, tal como indican estos mismos autores, otros trabajos como el de Gairín (1990, citado por Gil, Blanco y Guerrero, 2006) demuestran que la variable género no incide de modo significativo en las actitudes hacia las matemáticas ni tampoco se puede establecer una relación causal.

Primi, Busdraghi, Tomasetto, Morsanyi y Chiesi (2014) revisan la literatura existente en el campo afectivo relacionado con el aprendizaje de la matemática, enfatizando en los resultados según el sexo. De igual modo, Birgin, Baloglu, Çatlı lu y Gurbuz (2010) reportan que hay estudios que muestran niveles similares de ansiedad matemática entre hombres y mujeres. No obstante, otros investigadores como Else-Quest, Hyde y Linn (2010), Jain y Dowson (2009), McGraw, Lubienski y Strutchens (2006), Rubinstein, Bialik y Solar (2012) y Hembree (1990), señalan que las mujeres tienen niveles más altos de ansiedad matemática que los hombres con la consecuencia de que son menos propensas a buscar oportunidades de resolución de problemas y tienden a evitar actividades relacionadas con matemática.

De manera similar, las diferencias por género en la resolución de problemas no muestran un patrón de comportamiento estable en cuanto a que un grupo sea más eficaz que el otro, aunque algunos estudios revelan un mejor desempeño por parte de los hombres.

En el ámbito costarricense, se han encontrado diferencias en el nivel de ansiedad matemática por género en estudiantes de la educación secundaria (Meza, Agüero y Suárez, 2014; Corrales, 2014; Castillo y Picado, 2014) y en la educación superior (Mena, 2014). En ambos documentos se evidencia que las mujeres resultan más ansiosas a la hora de estudiar la matemática. Sin embargo, no se conocen estudios en el ámbito nacional sobre la actitud hacia la resolución de problemas, por lo que la presente investigación resulta pionera.

Sobre las causas de las diferencias encontradas entre hombres y mujeres en algunas de las variables de tipo emocional involucradas en el aprendizaje de la matemática, las investigaciones no han logrado arribar a resultados concluyentes. De acuerdo con Perina (2002), Reyes (1984) y Martin (2007), una de las posibles explicaciones podría ser que las mujeres informan con mayor sinceridad sobre sus experiencias de ansiedad matemática que los hombres, en el momento de responder a los instrumentos de medida. De acuerdo con los anteriores autores, las diferencias encontradas en el nivel de ansiedad matemática entre hombres y mujeres, por ejemplo, no se deberían necesariamente a que las mujeres sean más ansiosas, sino a que son más propensas a admitir su nivel de ansiedad.

Posición similar comparten Primi, Busdraghi, Tomasetto, Morsanyi y Chiesi (2014), quienes señalan que las diferencias podrían deberse a la forma en que se realiza la medición (medidas de auto-informe) y a que las mujeres están más dispuestas a reportar sus sentimientos que los hombres.

Es importante considerar que los resultados obtenidos por Ashcraft y Kirk (2001), citados por Tejedor, Santos, García-Orza, Carratalá y Navas (2009), muestran que las personas con ansiedad alta hacia la matemática no son intelectualmente menos capaces que los demás, pero si evidencian que ante tareas de tipo aritmético se consideran peores y evitan afrontarlas.

La resolución de problemas constituye, desde hace algunos años, un eje transversal imprescindible en el aprendizaje matemático, y por ello distintos países han incorporado de manera explícita la resolución de problemas en el currículo de la educación primaria y secundaria (Monje, Pérez-Tyteca y Castro, 2012). En Costa Rica, los programas de matemática aprobados por el Consejo Superior de Educación en 2012 establecen la resolución de problemas como la 
estrategia metodológica principal (Ministerio de Educación Pública, 2012).

Aunque la resolución de problemas es una actividad claramente cognitiva, los procesos involucrados en ella son particularmente susceptibles de la influencia del dominio afectivo (Mc Leod, 1989, citado por Gómez-Chacón, 2000). Así, el estudio del afecto es fundamental si queremos tener una visión completa de los aspectos involucrados en el proceso de resolución de problemas (Monje, Pérez-Tyteca y Castro, 2012).

En cuanto a la influencia de los aspectos afectivos en la resolución de problemas, especialmente en la educación secundaria, se encuentran pocos estudios en la literatura especializada, lo que contrasta con la cantidad creciente de investigaciones en otros ámbitos de la educación matemática. En un estudio pionero Gil, Blanco y Guerrero (2006) investigaron las influencias de las creencias, actitudes y emociones (afectividad) en estudiantes de tercer y cuarto curso de Educación Secundaria Obligatoria en España, cuando se enfrentan a la resolución de problemas de matemáticas. Los resultados obtenidos indican que el género es una de las variables que influye con evidencia de resultados más negativos para las mujeres.

Las actitudes hacia la resolución de problemas han sido consideradas como una componente de peso en las matemáticas (Castro, 2008). En este sentido, Mc Leod (1989), citado por Gil, Blanco y Guerrero (2006), señala que los procesos cognitivos implicados en la resolución de problemas son particularmente susceptibles al influjo del ámbito afectivo.

Uno de los pilares teóricos fundamentales de la investigación en educación matemática es el supuesto de que los factores emocionales son un elemento explicativo clave para interpretar el éxito en la solución de problemas matemáticos (Gil, Blanco y Guerrero, 2006).

Diversos autores también han puesto en evidencia que la afectividad juega un importante papel en el proceso de resolución de un problema de matemática (Callejo, 1994; Gil, Blanco y Guerrero, 2005; Gil, Blanco y Guerrero, 2006 y Molina, 2012). Ello se debe a que es frecuente experimentar sentimientos en el proceso de solución de problemas matemáti- cos. Tales sentimientos y emociones pueden impulsar la búsqueda de una solución o, por el contrario, bloquear el proceso debido al peso de las emociones negativas (Gil, Blanco y Guerrero, 2006).

Igualmente Tárraga (2008) encontró relación entre la actitud hacia la matemática y la ansiedad matemática con el rendimiento en la resolución de problemas matemáticos y Castro (2008), reporta que diversos estudios sugieren una correlación bastante alta entre la actitud hacia la matemática y la resolución de problemas con el rendimiento en esta disciplina, aunque no ha sido posible encontrar una relación de causalidad que muestre que el fracaso en resolver problemas se deba a una mala actitud.

La importancia de estudiar el nivel de actitud hacia la resolución de problemas matemáticos radica en que "las actitudes hacia la matemática, en sus diversas manifestaciones, pueden ser mejoradas mediante cambios en la predisposición de pensar respecto al objeto mediante sentimientos más positivos (afectividad)" (Mato y Muñoz, 2010, p. 27).

El efecto del estrés en el rendimiento fue ampliamente estudiado por los investigadores Yerkes y Dodson (1908), quienes encontraron que para alcanzar un comportamiento eficiente la motivación no debe llegar a un nivel muy bajo, pero tampoco demasiado alto. Esta afirmación, conocida como "Ley de Yerkes y Dodson", establece una relación entre la motivación y el estrés con el rendimiento.

El comportamiento se representa como una " $U$ " invertida, interpretada de la siguiente manera: a bajos o altos niveles de estrés se genera un bajo rendimiento. Al existir un valor óptimo de estrés, este ocasiona el máximo de rendimiento en el individuo.

En términos del proceso de enseñanza-aprendizaje, el docente debe conocer la "presión" óptima o exigencia hacia sus alumnos que le permita un óptimo rendimiento en su aprendizaje. El docente debe trabajar en una región segura de nivel de exigencia hacia sus alumnos, que no llegue a desestimular ni provocar un bajo rendimiento. Las expectativas además deben ser suficientes pero no exageradas, de manera tal que promuevan un aumento en el rendimiento de sus alumnos. 
El estudio de estas variables en la educación secundaria es especialmente importante porque, de acuerdo con Aschcraft (2005), citado por Primi et al. (2014), "El interés y la motivación declinan conforme el estudiante va creciendo, y la ansiedad matemática se piensa que se desarrolla en la educación secundaria, coincidiendo con la creciente dificultad del plan de estudios de matemáticas".

Una posible aproximación teórica sobre las consecuencias de la baja actitud de las y los estudiantes hacia la resolución de problemas, o de la alta ansiedad matemática, en su caso, se encuentra en la "Teoría de control atencional" (ACT, por sus siglas en inglés) (Eysenck, Santos, Derakshan y Gutiérrez - Calvo, 2007) que representa un importante desarrollo de la teoría de la eficiencia de procesamiento de Eysenck y Gutiérrez - Calvo (1992).

De acuerdo con esta teoría, la capacidad de la memoria de trabajo de las personas que sufren una situación estresante disminuye por la reducción de la capacidad del ejecutivo central de inhibir respuestas dominantes o automáticas cuando sea necesario, alternar entre diferentes estados mentales o múltiples tareas y actualizar representaciones de la memoria de trabajo. La teoría plantea que lo anterior provoca que la persona que enfrenta una situación estresante tienda a depender más de su atención enfocada en el estímulo que la amenaza, que de las habilidades necesarias para realizar la tarea.

Como consecuencia, de acuerdo con estos autores, las personas que sufren de ansiedad tienden a ser menos eficientes en la resolución de las tareas, porque los pensamientos poco relevantes a la tarea en desarrollo, como los generados por las preocupaciones, tendrán un efecto negativo al requerir de recursos atencionales que deberían ser empleados en el procesamiento de la tarea. Esta demanda de recursos se explica porque las personas ansiosas tratarán de mejorar su efectividad a expensas de su eficiencia.

\section{MATERIALES Y MÉTODOS}

La investigación desarrollada tuvo un enfoque cuantitativo, con un alcance descriptivo y se realizó con estudiantes de la educación media costarricense matriculados en el año 2014 en colegios académicos oficiales. Mediante una técnica de muestreo simple aleatorio, se seleccionaron cinco centros educativos de cada provincia y dentro de cada uno de ellos un grupo de cada nivel, integrando una muestra de 3271 estudiantes $(51.24 \%$ de mujeres y $48.76 \%$ de hombres). En la tabla 1 se muestra la distribución de la muestra por nivel educativo:

Tabla 1

Distribución de la muestra por nivel educativo

\begin{tabular}{lcc}
\multicolumn{1}{c}{ Nivel } & Frecuencia & Porcentaje \\
\hline Sétimo & 633 & 19,4 \\
\hline Octavo & 653 & 20,0 \\
\hline Noveno & 651 & 19,9 \\
\hline Décimo & 697 & 21,3 \\
\hline Undécimo & 637 & 19,5 \\
\hline Total & $\mathbf{3 2 7 1}$ & $\mathbf{1 0 0 , 0}$ \\
\hline
\end{tabular}

Como instrumento de medición se utilizó la "Escala de Actitud hacia la Resolución de Problemas Matemáticos" de Gil, Blanco y Guerrero (2006), por ajustarse a los propósitos de la investigación. Este instrumento consiste en un cuestionario tipo Likert integrado por nueve ítems, para los cuales existen 5 posibilidades de respuesta que van desde "Totalmente de acuerdo" a "Totalmente en desacuerdo", con la opción central o neutra "Indeciso".

Para evidenciar la validez del instrumento de medida se realizó un estudio del supuesto de unidimensionalidad mediante técnicas del análisis factorial exploratorio por ser la técnica más utilizada para estos propósitos (Jiménez y Montero, 2013), constatando el cumplimiento del criterio planteado por Carmines y Zeller (1979), citados por Burga (2006), el cual consiste en que el primer factor explique al menos el $40 \%$ de la varianza. La confiabilidad del instrumento se estudió mediante la técnica del Alfa de Cronbach.

Las hipótesis consideradas en la investigación, formuladas como hipótesis nulas, fueron las siguientes:

Hipótesis 1: No existen diferencias en la variable "actitud hacia la resolución de problemas matemáti$\cos ^{\prime \prime}$ por sexo.

Hipótesis 2: No existen diferencias en la variable "actitud hacia la resolución de problemas matemáti$\cos ^{\prime \prime}$ por nivel educativo. 


\section{RESULTADOS}

La investigación consideró tres variables: sexo, nivel educativo y actitud hacia la resolución de problemas matemáticos. En la tabla 2 se muestran los resultados obtenidos de la variable actitud hacia la resolución de problemas matemáticos.

Tabla 2. Clasificación del nivel de actitud hacia la resolución de problemas matemáticos

\begin{tabular}{lcc}
\multicolumn{1}{c}{ Categoría } & Frecuencia & Porcentaje \\
\hline Nivel muy bajo & 18 & 0,55 \\
\hline Nivel bajo & 631 & 19,29 \\
\hline Nivel medio & 1554 & 47,51 \\
\hline Nivel alto & 951 & 29,07 \\
\hline Nivel muy alto & 117 & 3,58 \\
\hline Total & $\mathbf{3 2 7 1}$ & $\mathbf{1 0 0 , 0 0}$ \\
\hline
\end{tabular}

Estos resultados indican que, aproximadamente, un $20 \%$ de los y las estudiantes presentan niveles de actitud hacia la resolución de problemas matemáticos baja o muy baja, y una actitud media aproximadamente del $48 \%$.

En la tabla 3 se muestran los resultados acerca del índice de discriminación obtenido para cada ítem del cuestionario, utilizando la correlación entre la puntuación obtenida en el ítem y la obtenida en el instrumento excluyendo la correspondiente al ítem, para no incrementar de manera artificial el valor de la correlación entre ambas puntuaciones (Lozano y De la Fuente-Solana, 2013, p. 11).

Tabla 3. Índice de discriminación de los ítems

\begin{tabular}{llc}
\multicolumn{1}{c}{ Ítems } & $\begin{array}{c}\text { Índice de } \\
\text { discriminación }\end{array}$ \\
\hline Ítem 1 & 0,412 \\
\hline Ítem 2 & 0,228 \\
\hline Ítem 3 & 0,374 \\
\hline Ítem 4 & 0,015 \\
\hline Ítem 5 & 0,342 \\
\hline Ítem 6 & 0,342 \\
\hline Ítem 7 & 0,149 \\
\hline Ítem 8 & 0,357 \\
\hline Ítem 9 & 0,132 \\
\hline
\end{tabular}

Se deduce entonces, siguiendo la clasificación de Lozano y De la Fuente-Solana (2013, p. 12), que solo los ítems 1, 3, 5, 6 y 8 tienen un índice de discriminación aceptable pues superan el valor de 0,3. De esta forma, conservando dichos ítems y volviendo a calcular el índice de discriminación, se obtienen los resultados que se muestran en la tabla 4 .

Tabla 4. Índice de discriminación de los ítems

\begin{tabular}{ccc}
\hline \multicolumn{2}{c}{ Ítems } & Índice de discriminación \\
\hline Ítem & 1 & 0,408 \\
\hline Ítem & 3 & 0,449 \\
\hline Ítem & 5 & 0,317 \\
\hline Ítem & 6 & 0,450 \\
\hline Ítem & 8 & 0,423 \\
\hline
\end{tabular}

Dado que todos los valores superan el valor de 0,3 , se puede considerar que muestran un adecuado nivel de discriminación. Consecuentemente, los análisis restantes se realizaron a partir de los valores arrojados por estos cinco ítems.

Con respecto a la confiabilidad del instrumento de medición, el valor del alfa de Cronbach obtenido fue de 0,76, razón por la cual, siguiendo el criterio de George y Mallery (1995), citados por Fernández (2003, p. 197), se tiene que el instrumento mostró un nivel aceptable de confiabilidad.

Para estudiar la unidimensionalidad del instrumento se calcularon los índices Kaiser-Meyer-Olkin (KMO) y de esfericidad de Bartlett, con los resultados se muestran en la tabla 5.

Tabla 5. KMO y prueba de esfericidad de Bartlett

\begin{tabular}{lll}
\hline \multicolumn{2}{l}{$\begin{array}{l}\text { Medida de adecuación muestral de } \\
\text { Kaiser-Meyer-Olkin }\end{array}$} & $\mathbf{0 , 6 9 7}$ \\
\hline $\begin{array}{l}\text { Prueba de } \\
\text { esfericidad de }\end{array}$ & $\begin{array}{l}\text { Chi-cuadrado } \\
\text { Bartlett }\end{array}$ & 16126,773 \\
\cline { 2 - 3 } & Gl & 55 \\
\cline { 2 - 3 } & Sig. & 0,000 \\
\hline
\end{tabular}

El valor de 0,697 para el índice KMO indica una adecuación muestral regular, de acuerdo con las recomendaciones dadas por Kaiser en 1974, citado por Frías-Navarro y Pascual (2012). Por otra parte, el valor $p<0,05$ en el índice de Bartlett indica que es 
Figura 1. Histograma para sexo masculino

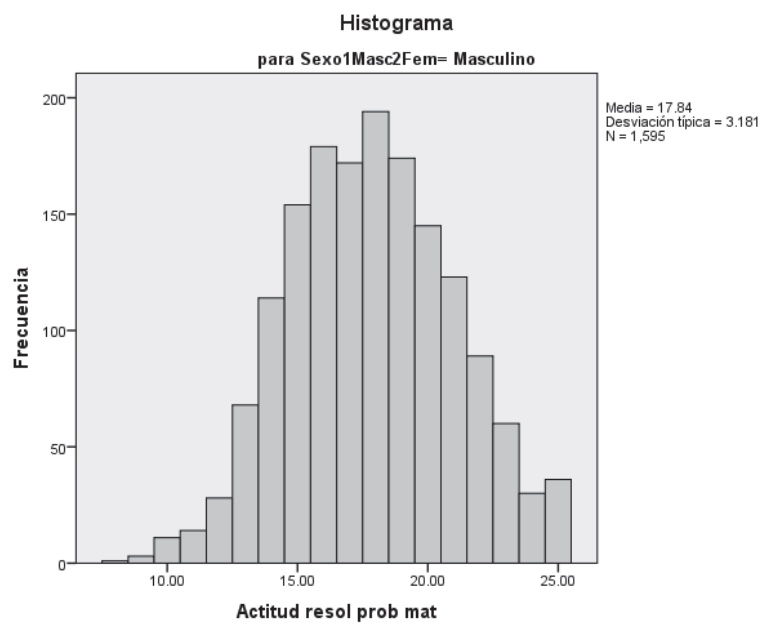

Figura 3. Gráfico Q-Q para sexo masculino

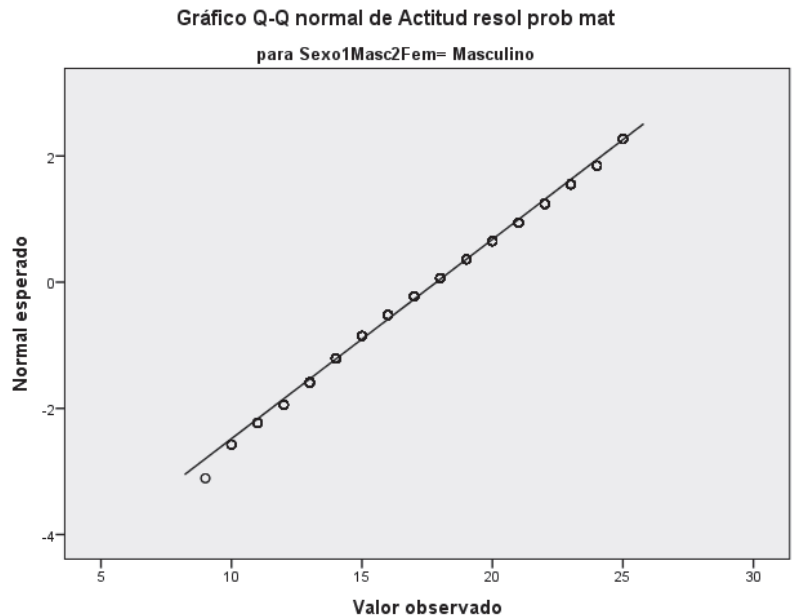

adecuado desarrollar un análisis factorial con estos datos. El análisis factorial arrojó los resultados que se muestran en la tabla 6.

Tabla 6. Resultados del análisis factorial

\begin{tabular}{|c|c|c|c|}
\hline \multirow[b]{2}{*}{$\begin{array}{c}\text { Compo- } \\
\text { nente }\end{array}$} & \multicolumn{3}{|c|}{ Autovalores iniciales } \\
\hline & Total & $\begin{array}{c}\% \text { de la } \\
\text { varianza }\end{array}$ & $\begin{array}{c}\% \\
\text { acumulado }\end{array}$ \\
\hline 1 & 2,051 & 41,011 & 41,011 \\
\hline 2 & 1,007 & 20,142 & 61,153 \\
\hline 3 & 0,733 & 14,658 & 75,811 \\
\hline 4 & 0,673 & 13,456 & 89,267 \\
\hline 5 & 0,537 & 10,733 & 100,000 \\
\hline
\end{tabular}

Figura 2. Histograma para sexo femenino

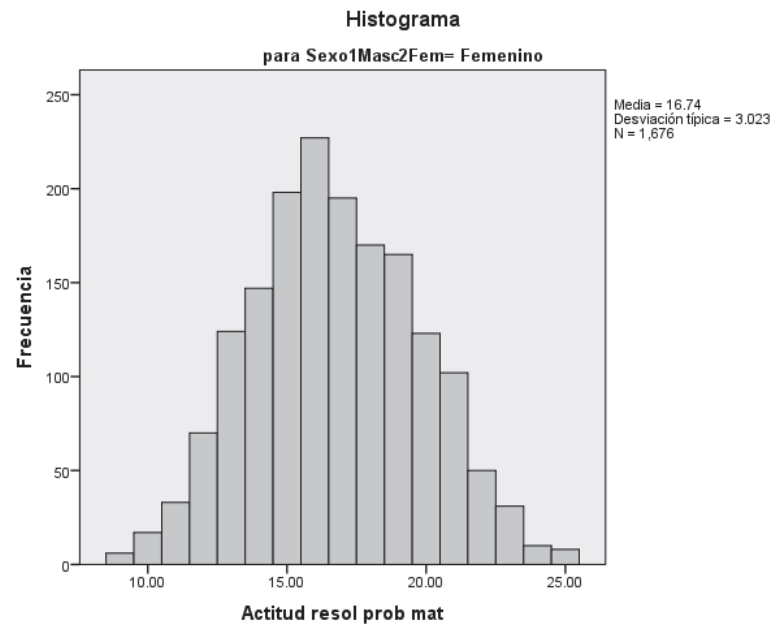

Figura 4. Gráfico $Q-Q$ para sexo femenino

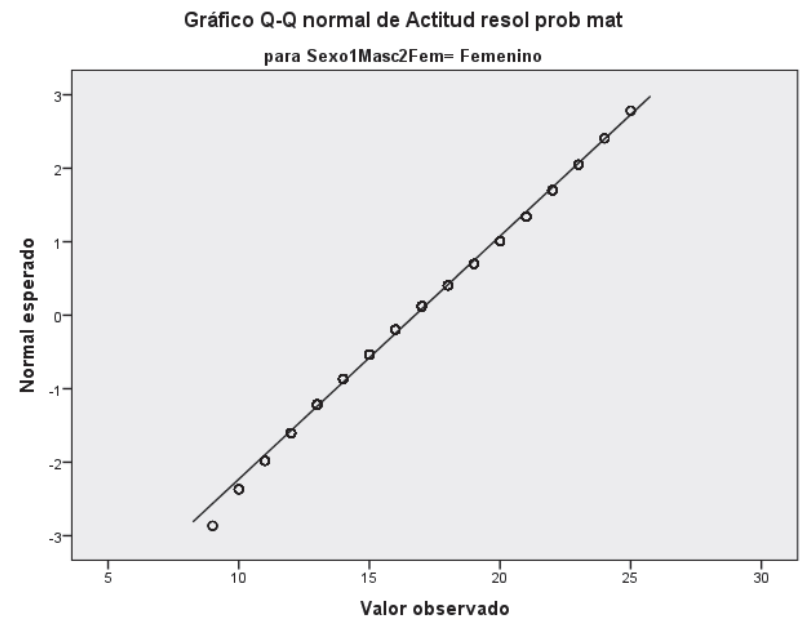

En esta tabla se observa que el primer autovalor explica el 41,011\% de la varianza total, razón por la que se puede tener por cumplido el criterio de Carmines y Zeller (1979), citado por Burga (2006), con lo cual se concluye que existe evidencia de la unidimensionalidad del instrumento.

Para el contraste de la hipótesis 1, se utilizó como hipótesis nula que "no existen diferencias en la variable actitud hacia la resolución de problemas matemáticos por sexo" y como hipótesis alternativa que "sí existen diferencias significativas en la variable actitud hacia la resolución de problemas matemáticos por sexo". 
Como la hipótesis incluye solo dos categorías, la comparación de medias entre dos grupos independientes se abordó con el test $t$ de Student, previo análisis del cumplimiento de las condiciones exigibles al utilizar métodos paramétricos.

Para analizar si los datos seguían una distribución normal se realizó una exploración gráfica y se aplicó la prueba de normalidad de Kolmogorov-Smirnov.

La exploración gráfica se fundamentó en los gráficos que se muestran en las figuras 1 a 4 , las cuales sugieren la normalidad en ambas muestras involucradas en la hipótesis.

Con los gráficos mostrados en las figuras 3 y 4 se puede observar qué tan cerca está la distribución de un conjunto de datos a alguna distribución ideal o comparar la distribución de dos conjuntos de datos. Si los datos proceden de una distribución normal los puntos aparecen agrupados en torno a la línea recta esperada (Castillo y Alzamora, 2011).

Considerando la evidencia de normalidad que se desprende de los gráficos de las figuras 1 a 4, más el hecho de que cada muestra tenía más de 100 casos se puede asumir la normalidad (Aguayo, 2004).

Los datos de la tabla 7 para la prueba de Levene permiten asumir la homogeneidad de las varianzas.

Tabla 7. Prueba de muestras independientes

\begin{tabular}{ccc}
\hline & \multicolumn{2}{c}{$\begin{array}{c}\text { Prueba de Levene para la } \\
\text { igualdad de varianzas }\end{array}$} \\
\cline { 2 - 3 } & $\mathbf{F}$ & Sig. \\
\hline $\begin{array}{c}\text { Actitud resolución } \\
\text { de problemas }\end{array}$ & 3,446 & 0,064 \\
\hline
\end{tabular}

En la tabla 8 se muestran los resultados de la aplicación de la prueba t de Student.
Tabla 8. Prueba t de Student

\begin{tabular}{|c|c|c|c|c|}
\hline \multicolumn{5}{|c|}{ Prueba de muestras independientes } \\
\hline \multirow{2}{*}{\multicolumn{2}{|c|}{$\mathrm{T}$}} & \multicolumn{3}{|c|}{$\begin{array}{c}\text { Prueba T para la igualdad de } \\
\text { medias }\end{array}$} \\
\hline & & gl & $\begin{array}{c}\text { Sig. } \\
\text { (bilateral) }\end{array}$ & \\
\hline \multirow[t]{2}{*}{$\begin{array}{c}\text { Actitud } \\
\text { resol. } \\
\text { probl. }\end{array}$} & $\begin{array}{c}\text { Se han } \\
\text { asumido } \\
\text { varianzas } \\
\text { iguales }\end{array}$ & 10,082 & 3269 & 0,000 \\
\hline & $\begin{array}{c}\text { No se han } \\
\text { asumido } \\
\text { varianzas } \\
\text { iguales }\end{array}$ & 10,069 & 3236,485 & 0,000 \\
\hline
\end{tabular}

Dado que el valor de la significación estadística es menor que 0,05, asumiendo varianzas iguales según la prueba de Levene, lo que corresponde es rechazar la hipótesis nula y aceptar la hipótesis alternativa. En otros términos, existe evidencia estadística para apoyar la hipótesis de que existen diferencias en la variable actitud hacia la resolución de problemas matemáticos por sexo.

La medida del tamaño del efecto de esta diferencia en el nivel de actitud por sexo se calculó utilizando la $d$ de Cohen, resultado en un valor $d=0,35$, lo que permite clasificar la magnitud del efecto, de acuerdo con los criterios de Cohen, entre baja y moderada.

Para el contraste de la hipótesis 2, se utilizó como hipótesis nula que "no existen diferencias en la variable actitud hacia la resolución de problemas matemáticos por nivel educativo" y como hipótesis alternativa que "sí existen diferencias significativas en la variable actitud hacia la resolución de problemas matemáticos por nivel educativo".

Dado que la variable se analiza en cinco categorías, se aplicó el análisis de la varianza (ANOVA), la cual también requiere de la normalidad de la distribución de los datos en cada una de las muestras involucradas en el contraste y de la homogeneidad de varianzas.

Debido a que cada una de las series de datos en comparación tiene más de 100 casos, se asumió la normalidad (Aguayo, 2004) y para la homogeneidad de varianzas los resultados de la prueba de Levene se muestran en la tabla 9. 
Resolución de problemas matemáticos en la educación media costarricense: un estudio acerca de la actitud

Tabla 9. Prueba de homogeneidad de varianzas

\begin{tabular}{cccc}
\hline Estadístico de Levene & gl1 & gl2 & Sig. \\
\hline 1,930 & 4 & 3266 & 0,103 \\
\hline
\end{tabular}

Como el valor del estadístico de Levene tiene un índice de significancia de 0,103, esto es p>0,05, se puede asumir la homogeneidad de varianzas.

Los resultados de la prueba ANOVA se presentan en la tabla 10.

Tabla 10. Resultados de la prueba ANOVA

\begin{tabular}{cccccc}
\hline & $\begin{array}{c}\text { Suma de } \\
\text { cuadrados }\end{array}$ & Gl & $\begin{array}{c}\text { Media } \\
\text { cuadrática }\end{array}$ & F & Sig. \\
\hline $\begin{array}{c}\text { Inter- } \\
\text { grupos }\end{array}$ & 508,218 & 4 & 127,054 & 9,436 & 0,000 \\
\hline $\begin{array}{l}\text { Intra- } \\
\text { grupos }\end{array}$ & 43977,365 & 3266 & 13,465 & & \\
\hline \begin{tabular}{c} 
Total \\
\hline
\end{tabular} & 44485,583 & 3270 & & & \\
\hline
\end{tabular}

Como el valor de la significancia es $\mathrm{p}<0,05$, se rechaza la hipótesis nula y se acepta la hipótesis alternativa. En otras palabras, hay evidencia estadística de la existencia de diferencias significativas en la variable actitud hacia la resolución de problemas matemáticos según el nivel educativo que cursa el o la estudiante.

Los resultados del análisis ANOVA permiten establecer la evidencia estadística de que existen diferencias en el nivel de actitud hacia la resolución de problemas matemáticos entre algunos de los niveles educativos de la educación media, pero falta determinar entre cuáles niveles es que se dan tales diferencias, lo que puede hacerse con las denominadas pruebas post hoc o a posteriori, de las cuales se utilizó la prueba de Scheffé.
La tabla 11 muestra una clasificación de los niveles educativos basada en el grado de parecido que existe entre sus medias, a partir del método de Scheffé.

Tabla 11. Subconjuntos homogéneos

\begin{tabular}{lccc}
\multicolumn{1}{c}{ Nivel } & $\mathbf{1}$ & $\mathbf{2}$ & $\mathbf{3}$ \\
\hline Décimo & 15.1506 & & \\
\hline Undécimo & 15.5918 & 15.5918 & \\
\hline Octavo & & 15.9510 & 15.9510 \\
\hline Noveno & & 15.9816 & 15.9816 \\
\hline Sétimo & & & 16.2891 \\
\hline
\end{tabular}

En la tabla 11 se observa que se forman tres subconjuntos homogéneos. En el primer subconjunto no existe diferencia estadísticamente significativa en el nivel promedio de la actitud hacia la resolución de problemas para décimo y undécimo. De igual manera, no hay diferencias significativas entre undécimo, octavo y noveno. Finalmente, no hay diferencias entre sétimo, octavo y noveno. Además, décimo difiere de sétimo, octavo y noveno, y undécimo de sétimo.

El estudio de la diferencia en el nivel de actitud hacia la resolución de problemas matemáticos se profundiza al efectuarlo considerando el nivel que cursaban los estudiantes. En la Tabla 12 se muestran los valores de la media y la desviación estándar para cada género cuando se hacen los cálculos por cada nivel educativo, además de los valores $T$ y de la significancia para contrastar la hipótesis nula en cada nivel de que no hay diferencias en el la actitud hacia la resolución de problemas matemáticos por género.

Los datos de la Tabla 12 permiten concluir que las diferencias por género en la actitud hacia resolución de problemas matemáticos se presentan en cada uno de los cinco niveles.

Tabla 12. Estadísticos para comparar por sexo y nivel

\begin{tabular}{ccccccc}
\hline Nivel & Media hombres & DS & Media mujeres & DS & T & p-value \\
\hline Sétimo & 16.8843 & 3.78298 & 15.6115 & 3.64451 & 4.296 & 0.000 \\
\hline Octavo & 16.6821 & 3.50420 & 15.2310 & 3.57943 & 5.234 & 0.000 \\
\hline Noveno & 16.6069 & 3.71157 & 15.4792 & 3.65912 & 3.883 & 0.000 \\
\hline Décimo & 15.9884 & 3.72529 & 14.3295 & 3.62870 & 5.955 & 0.000 \\
\hline Undécimo & 16.2977 & 3.36509 & 14.9675 & 3.42215 & 4.934 & 0.000 \\
\hline
\end{tabular}




\section{DISCUSIÓN Y CONCLUSIONES}

Los resultados sugieren que solo un $20 \%$ de los y las estudiantes manifiestan una actitud baja o muy baja hacia la resolución de problemas matemáticos. Pese a esto, un $50 \%$ de la muestra presentó una actitud media ante la resolución de problemas matemáticos.

Estos resultados deberían servir para que los y las docentes, y las propias autoridades educativas, se enfoquen en acciones concretas encaminadas a mejorar la actitud de los y las estudiantes hacia la resolución de problemas. Como plantea McLeod (1989), citado por Monje, Pérez-Tyteca y Castro (2012), los estudiantes que tienen experiencias positivas con la resolución de problemas no rutinarios, con frecuencia pueden desarrollar actitudes de curiosidad y entusiasmo en torno a ellos.

Desde esta perspectiva, parece recomendable que los docentes propongan problemas matemáticos a sus estudiantes con una dificultad incremental, iniciando con problemas de fácil comprensión que les ofrezcan una mayor probabilidad de ser resueltos exitosamente. De esta manera se promoverán experiencias positivas en la resolución de problemas que redunden en una mejora de la actitud. En otras palabras, la cuidadosa selección de los primeros problemas que se proponga resolver a las y los estudiantes, podría ser un elemento clave para mejorar el nivel de actitud que manifiesten sobre la resolución de problemas.

La investigación también devela que existen diferencias entre hombres y mujeres en el nivel de actitud hacia la resolución de problemas, con resultados menos favorables para las mujeres, lo que coincide con la investigación de Gil, Blanco y Guerrero (2006).

Aunque las diferencias en aspectos afectivos relacionados con el aprendizaje de la matemática no se detectan en todas las investigaciones (Gairín, 1990, citado por Gil, Blanco y Guerrero, 2006), resulta relevante resaltar que en el tema de la ansiedad matemática también se han detectado diferencias según el sexo en la educación media costarricense, con resultados menos favorables para las mujeres (Meza,
Agüero y Suárez, 2014; Corrales, 2014; Castillo y Picado, 2014).

No obstante, dado que el tamaño del efecto de las diferencias es bajo, tanto en las investigaciones sobre ansiedad matemática como sobre la actitud hacia la resolución de problemas, conviene mirar con precaución tales diferencias y valorar, tal como sugieren González, Fernández, García, Suárez, Fernández, Tuero y Da Silva (2012), que podrían estar explicadas por la presencia de otras variables que no han sido consideradas en la investigación. La literatura sobre el tema no permite establecer posibles causas que expliquen estas diferencias, existiendo un cierto consenso en la actualidad acerca de que podrían deberse a efectos propios de la medición, generados debido a que por razones de tipo cultural las mujeres tienden a responder con mayor sinceridad que los hombres (Castro, 2008).

Otro de los resultados obtenidos muestra la existencia de diferencias significativas en la variable actitud hacia la resolución de problemas matemáticos según el nivel educativo a que pertenecen las y los estudiantes, mostrando mejores niveles de actitud en los niveles del tercer ciclo y menores en los del cuarto ciclo. Esta situación, aunado al hecho de que son estos niveles los que muestran los mayores niveles de ansiedad matemática (Meza, Agüero y Suárez, 2014), resulta preocupante por cuanto los niveles menos favorables se presentan precisamente con los y las estudiantes más próximos a escoger carrera universitaria.

El desconocimiento de las posibles causas que expliquen las diferencias detectadas entre los estudiantes según su sexo en la actitud hacia la resolución de problemas dificulta el diseño de actividades de intervención educativa orientadas a mejorar la actitud de las mujeres.

No obstante, los hallazgos de la investigación pueden servir para alertar a los docentes de matemática de la educación media y a otros actores educativos, como los orientadores o los directores de los colegios, sobre la necesidad de procurar programas de intervención educativa que mejoren los niveles de actitud hacia la resolución de problemas 
matemáticos de los y las estudiantes de la educación diversificada en general.

Considerando que algunas investigaciones (Tapia y Marsh, 2004, citados por Primi et. al., 2014), que muestran que se presentan correlaciones negativas entre niveles de ansiedad matemática y las actitudes hacia la matemática, cabe esperar que la actitud hacia la resolución de problemas matemáticos también se correlacione en forma negativa con la actitud hacia la matemática, siendo esta una línea de investigación que se sugiere a partir de los resultados de la investigación.

Los resultados sugieren también, de manera similar que en Meza, Agüero y Suárez (2014), dos líneas de investigación adicionales: profundizar en las causas que podrían explicar las diferencias detectadas en el nivel de actitud hacia la resolución de problemas matemáticos por sexo o descartar su existencia y las que puedan explicar por qué disminuye el nivel de actitud cuando los estudiantes llegan a la Educación Diversificada.

\section{AGRADECIMIENTOS}

Se agradece el apoyo de la Vicerrectoría de Investigación y Extensión del Instituto Tecnológico de Costa Rica para el desarrollo de la investigación (proyecto núm. 5402-1440-4401) y a los colegios participantes en la investigación.

\section{REFERENCIAS BIBLIOGRÁFICAS}

Aguayo, M. (2004). Cómo realizar "paso a paso" un contraste de hipótesis con SPSS para Windows y alternativamente con EPIINFO y EPIDAT: (II) Asociación entre una variable cuantitativa y una categórica (comparación de medias entre dos o más grupos independientes). Recuperado de http://www.fabis.org/html/ archivos/docuweb/contraste_hipotesis_2r.pdf_el 12 de octubre de 2014.

Birgin, O., Balo lub, M., Çatı $\square l u$, H., Gürbüz, R. (2010). An investigation of mathematics anxiety among sixth through eighth grade students in Turkey. Learning and individual differences. Journal of Psychology and Education, 20 (6), 654-658.

Burga, A. (2006). La unidimensionalidad de un instrumento de medición: perspectiva factorial. Revista de Psicología de la PUCP. XXIV (1), 53-80.

Callejo, M. (1994). Un club matemático para la diversidad. Madrid, Narcea.
Castillo, H. y Picado, A. (2014) Estudio de la ansiedad matemática en estudiantes de colegios técnicos de la educación media costarricense. Tesis de licenciatura en la Enseñanza de la Matemática asistida por Computadora no publicada. Instituto Tecnológico de Costa Rica.

Castillo, J. \& Alzamora, L. (2011). Estadística para la tesis de posgrado. Londres: LULU International.

Castro, E. (2008). Resolución de problemas: ideas, tendencias e influencias en España. En R. Luengo, B. Gómez, M. Camacho y B. Lorenzo (Eds.). Investigación en Educación Matemática XII (pp. 113-140). Badajoz: Sociedad Española de Investigación en Educación Matemática, SEIEM.

Corrales, J. (2014). Estudio del nivel de ansiedad matemática en estudiantes de tres colegios académicos nocturnos costarricenses. Tesis de licenciatura en la Enseñanza de la Matemática asistida por Computadora no publicada, Instituto Tecnológico de Costa Rica.

Else-Quest, N., Shibley Hyde, J. y Linn, M. (2010). Cross-National Patterns of Gender Differences in Mathematics: A MetaAnalysis. Psychological Bulletin American Psychological Association, 136 (1), 103-127.

Eysenck, M., Santos, R., Derakshan, N. y Gutiérrez - Calvo, M. (2007). Anxiety and cognitive performance: attentional control theory. Emotion, 7 (2), 336-353.

Eysenck, M. W. y Gutiérrez - Calvo, M. (1992). Anixiety and performance: The processing efficiency theory. Cognition and Emotion, 6, 409-434.

Fernández, M. (2003). Modelo de comportamiento de la organización virtual: una aplicación empírica a los sistemas de franquicia (tesis doctoral). Universidad de las Palmas de Gran Canaria, España. Recuperado de: http://www.eumed.net/tesisdoctorales/2006/mfm/2f.htm el 4 de enero de 2015

Frías-Navarro, D. \& Pascual, M. (2012). Prácticas del análisis factorial exploratorio (afe) en la investigación sobre Conducta del consumidor y marketing. Suma Psicológica, 19 (1), 45-58.

Gil, N., Blanco, L. \& Guerrero, E. (2005). El dominio afectivo en el aprendizaje de las matemáticas. Una revisión de sus descriptores básicos. Revista Iberoamericana de Educación Matemática, 2, 15-32.

Gil, N., Blanco, L. y Guerrero, B. (2006). El papel de la afectividad en la resolución de problemas matemáticos. Revista de educación (340), 551-569.

Gómez-Chacón, I. (2000). Matemática Emocional: Los afectos en el aprendizaje matemático. Madrid: Narcea, S. A. Ediciones.

González, J., Fernández, M., García, T., Suárez, N., Fernández, E., Tuero, E. \& Da Silva, E. (2012). Diferencias de género en actitudes hacia las matemáticas en la enseñanza obligatoria. Revista Iberoamericana de Psicología y Salud, 3 (1), 55-73. Recuperado de: http://www.redalyc.org/articulo.oa?id=245122736004 el 3 de enero de 2015.

Guerrero, E.; Blanco, L.J. \& Castro, F. (2001). Trastornos emocionales ante la educación matemática. En García, J.N. (Coord.). 
Aplicaciones de Intervención Psicopedagógica. Pirámide, 229237.

Hembree, R. (1990). The Nature, Effects, and Relief of Mathematics Anxiety. Journal for Research in Mathematics Education (21), 1, 33-46.

Hernández, G. (2011). Estado del arte de creencias y actitudes hacia las matemáticas. Cuadernos de Educación y Desarrollo, 3 (24). Recuperado de: http://www.eumed.net/rev/ced/24/ghs.htm el 18 de agosto de 2014.

Jain, S. y Dowson, M. (2009). Mathematics anxiety as a function of multidimensional selfregulation and self-efficacy. Contemporary Educational Psychology, 34 (3), 240-249.

Jiménez, K. \& Montero, E. (2013). Aplicación del modelo de Rasch, en el análisis psicométrico de una prueba de diagnóstico en matemática. Revista digital Matemática, Educación e Internet, $13(1), 1-23$.

Lozano, L. \& De la Fuente-Solana, E. (2013). Diseño y validación de cuestionarios. En Pantoja-Vallejo (coordinador). Manual básico para la realización de tesinas, tesis y trabajos de investigación. España: Editorial EOS.

Mato, M. D. y Muñoz, J. M. (2010). Efectos generales de las variables actitud y ansiedad sobre el rendimiento en matemáticas en alumnos de ESO. Implicaciones para la práctica educativa. Ciencias psicológicas, IV (1), 27-40.

Martín, I. M. (2007). Estrés académico en estudiantes universitarios. Apuntes de Psicología, 25 (1), 87-99.

McGraw, R., Lubienski, S. T. y Strutchens, M. E. (2006). A closer look at gender in NAEP mathematics achievement and affect data: Intersections with achievement, race/ethnicity, and socioeconomic status. Journal for Research in Mathematics Education, 37 (2), 129-150.

Mena, J. (2014). Estudio de la ansiedad matemática en los cursos Matemática General, Cálculo Diferencial e Integral y Ecuaciones Diferenciales del Instituto Tecnológico de Costa Rica en el I Semestre 2013. Tesis de Licenciatura en la Enseñanza de la Matemática asistida por Computadora no publicada. Instituto Tecnológico de Costa Rica.

Meza, G., Agüero, E. y Suárez, Z. (2014). ESAM: Estudio de la ansiedad matemática en la educación media. Informe final de proyecto de investigación del Instituto Tecnológico de Costa Rica. Recuperado de http://repositoriotec.tec.ac.cr/handle/2238/4733 el 15 de mayo de 2015.

Ministerio de Educación Pública. (2012). Reforma curricular en ética, estética y ciudadanía programas de estudio de matemáticas I y II Ciclo de la Educación Primaria, III Ciclo de Educación General Básica y Educación Diversificada. Costa Rica. Recuperado de: www.mep.go.cr/sites/default/files/programadeestudio/ el 16 de noviembre de 2014.

Molina, E. (2012). Factores de la actitud y ansiedad al aprendizaje de la matemática en estudiantes adolescentes de la ciudad de Milagro. La relación de la estructura familiar y el rendimiento académico. Unión: Revista Iberoamericana de Educación Matemática, 29, 109-120.

Monje, J., Pérez-Tyteca, P. \& Castro, E. (2012). Resolución de problemas y ansiedad matemática: profundizando en su relación. Unión: Revista Iberoamericana de Educación Matemática, 32 , 45-62.

Núñez-Peña, M.I.; Suárez-Pellicioni, M. y Bono, R. (2013). Effects of math anxiety on student success in higher education. International Journal of Educational Research, 58, 36-43.

Palacios, A., Arias, V. y Arias, B. (2014). Las actitudes hacia las matemáticas: construcción y validación de un instrumento para su medida. Revista de Psicodidáctica de la Universidad del país Vasco, 19 (1), 67-91. Recuperado de: http://www.ehu.eus/ojs/index.php/psicodidactica el 5 de julio de 2015.

Pérez-Tyteca, P. (2012). La ansiedad matemática como centro de un modelo causal predictivo de la elección de carreras. Disertación doctoral no publicada, Universidad de Granada. España.

Perina, K. (2002). The sum of all fears. Psychology Today, 35 (6), 19.

Primi, C., Busdraghi, C., Tomasetto, C., Morsanyi, K. y Chiesi, F. (2014). Measuring math anxiety in Italian college and high school students: validity, reliability and gender invariance of the Abbreviated Math Anxiety Scale (AMAS). Learning and Individual Differences, 34, 51-56.

Reyes, L. H. (1984). Affective variables and mathematics education. The Elementary School Journal, 84 (5), 558-581.

Rubinsten, O., Bialik, N. y Solar, Y. (2012). Exploring the relationship between math anxiety and gender through implicit measurement. Frontiers in Human Neuroscience (6), 1-11.

Tárraga, R. (2008). Relación entre rendimiento en solución de problemas y factores afectivo-motivacionales en alumnos con y sin dificultades del aprendizaje. Apuntes de Psicología, 26 (1), 143-148.

Tejedor, B., Santos, M. A., García-Orza, J., Carratalá, P., \& Navas, M. (2009). Variables explicativas de la ansiedad frente a las matemáticas: un estudio de una muestra de $6^{\circ}$ de primaria. Anuario de psicología, 40 (3), 345-355.

Yerkes, R., Dodson, J. (1908). The relation of strenght of stimulus to rapidity of habit-formation. Journal of Comparative Neurology and Psychology, 18, 459-482. 